\title{
High resolution 2D image upconversion of incoherent light
}

\author{
Dam, Jeppe Seidelin; Pedersen, Christian; Tidemand-Lichtenberg, Peter
}

Published in:

Proceedings of SPIE - The International Society for Optical Engineering

Link to article, DOI:

$10.1117 / 12.873790$

Publication date:

2011

Document Version

Publisher's PDF, also known as Version of record

Link back to DTU Orbit

Citation (APA):

Dam, J. S., Pedersen, C., \& Tidemand-Lichtenberg, P. (2011). High resolution 2D image upconversion of incoherent light. Proceedings of SPIE - The International Society for Optical Engineering, 7917, 791713. https://doi.org/10.1117/12.873790

\section{General rights}

Copyright and moral rights for the publications made accessible in the public portal are retained by the authors and/or other copyright owners and it is a condition of accessing publications that users recognise and abide by the legal requirements associated with these rights.

- Users may download and print one copy of any publication from the public portal for the purpose of private study or research.

- You may not further distribute the material or use it for any profit-making activity or commercial gain

- You may freely distribute the URL identifying the publication in the public portal

If you believe that this document breaches copyright please contact us providing details, and we will remove access to the work immediately and investigate your claim. 


\title{
High resolution 2-D image upconversion of incoherent light
}

\author{
Jeppe Seidelin Dam, Christian Pedersen* and Peter Tidemand-Lichtenberg \\ DTU Fotonik, Technical University of Denmark, DK-4000 Roskilde, Denmark \\ *Corresponding author: chrp@fotonik.dtu.dk
}

\begin{abstract}
An optimized method for continuous wave 2-dimensional (2-D) upconversion of incoherent or thermal light is demonstrated and quantified. Using standard resolution targets a resolution of 200x1000 pixels is obtained. The suggested method is viewed in scope of modern CCD cameras operating in the near infrared (NIR) portion of the electromagnetic spectrum. The key is optimization of the upconversion process. This include Quasi-Phase-Matching leading to higher effective nonlinearities and elimination of walk-off, an intra-cavity design enhancing the upconversion process, and finally the use of modern NIR CCD detectors. Furthermore, we discuss the exceptionally good depth of field possible for imaging systems based on the proposed method.
\end{abstract}

Keywords: Upconversion, thermal imaging, infrared imaging, Sum frequency generation.

\section{INTRODUCTION}

An optimized method for continuous wave 2-dimensional (2-D) upconversion of incoherent or thermal light is demonstrated and quantified. Using standard resolution targets, a resolution of 200x1000 pixels is obtained. The suggested method is viewed in scope of modern CCD cameras operating in the near infrared (NIR) portion of the electromagnetic spectrum. Upconversion based on Sum Frequency Generation (SFG) was already considered in the 1960'ties [1-2] as a possible means for detection of light at wavelength ranges inaccessible for direct detection due to lack of efficient detector materials. Such wavelength regions include the mid- and far-infrared. However, inefficiency in the upconversion process was prohibitive, and consequently image upconversion was abandoned [3] as a means to facilitate infrared imaging. Recently we have demonstrated approximately $25 \%$ quantum efficiency upconversion of 2-D images using coherent illumination of the object [4].

In this work, we extend the method used for coherent illumination case to include incoherently illuminated objects, i.e. objects emitting incoherent light. The key is an optimization of each step of the upconversion process. This include Quasi-Phase-Matching leading to higher effective nonlinearities and the elimination of walk-off, an intra-cavity design enhancing the upconversion process, and finally the use of modern NIR CCD detectors. All in all, the efficiency of the upconversion process is increased by $10^{5}$ compared to prior designs. An additional advantage compared to state-of-theart thermal cameras is the possibility to tailor the spectral response leading to functional spectral imaging.

Nonlinear Frequency Generation and Conversion: Materials, Devices, and Applications X, edited by Konstantin L. Vodopyanov, Proc. of SPIE Vol. 7917, 791713 - (c) 2011 SPIE

CCC code: $0277-786 X / 11 / \$ 18 \cdot$ doi: $10.1117 / 12.873790$ 


\section{DESCRIPTION AND EXPERIMENTAL SET-UP}

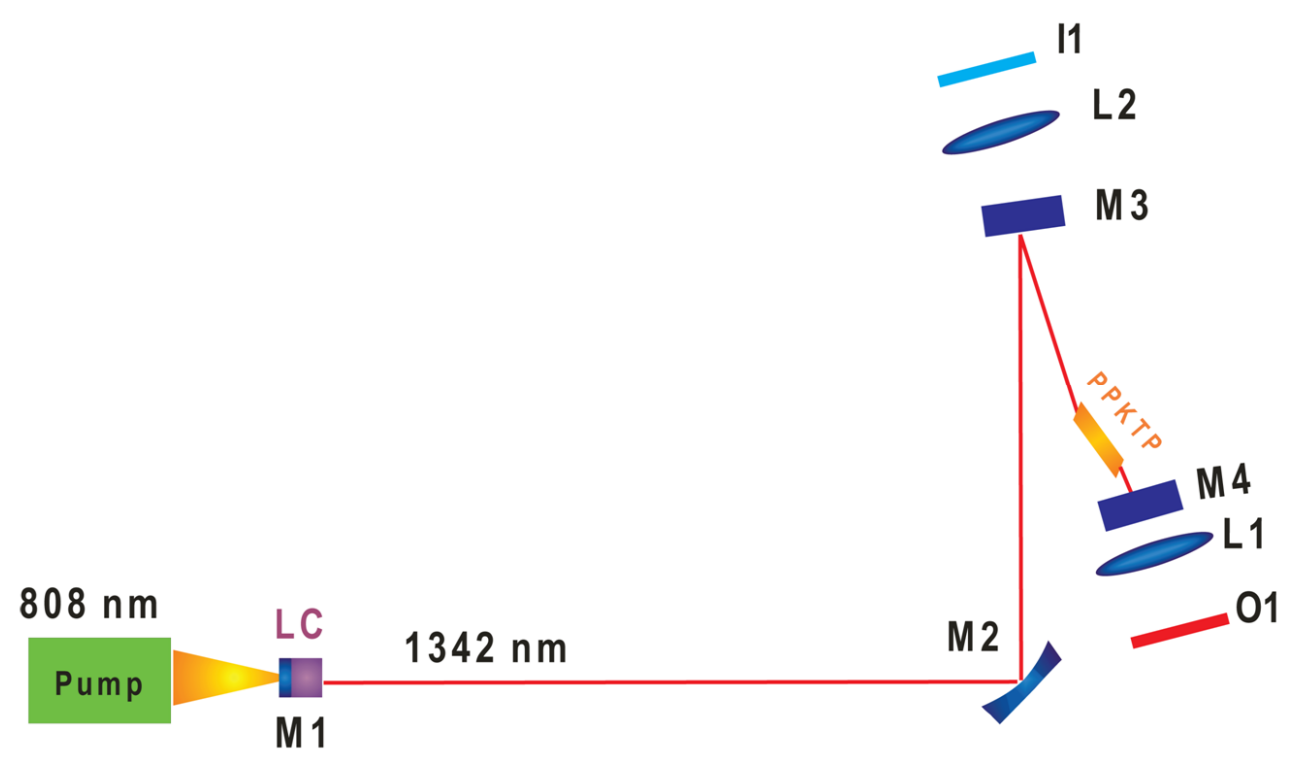

Figure 1. Experimental set-up.

The experimental set-up consists of three parts. See Fig. 1. A nonlinear medium designed for Sum Frequency Generation (SFG). Note that one of the two interacting fields in the SFG process is incoherent light emitted from an external light source (see Fig. 2). The other field involved is a high intensity Gaussian laser beam. This particular form of SFG is also referred to as upconversion. A $1 \times 3 \times 10 \mathrm{~mm}$ periodically poled KTP crystal with a poling period of $7.2 \mu \mathrm{m}$ is used, supporting SFG of a $1342 \mathrm{~nm}$ laser beam and incoherent light at $766 \mathrm{~nm}$. The generated field will be centered close to $488 \mathrm{~nm}$. The second part is a High Finesse, diode pumped, $1342 \mathrm{~nm} \mathrm{Nd:YAG} \mathrm{laser} \mathrm{including} \mathrm{the} \mathrm{pp-KTP} \mathrm{crystal} \mathrm{as} \mathrm{an}$ intra-cavity component (see Fig.1). The power of the upconverting beam is approximately 5 Watts. Note that the purpose of the enhancement cavity is merely to increase conversion efficiency. The (simple) astigmatic cavity design is designed to support a wider beam waist along the $3 \mathrm{~mm}$ direction of the pp-KTP so as to make the full use of the crystals aperture; The bigger the beam waist the better resolution in the imaging process. The third element is the imaging optics. The lens optics is designed as a 4-f system with the nonlinear medium located at the Fourier plane. This particular set-up has several advantages over more elaborate designs. First it allows us to derive a simple expression for the imaging process. Secondly, it is infinity corrected which effectively cancels image aberrations caused by the finite length of the non-linear crystal. Thirdly, it gives us a simple pictorial approach to the imaging process applying Huygens - Fresnels principle. 


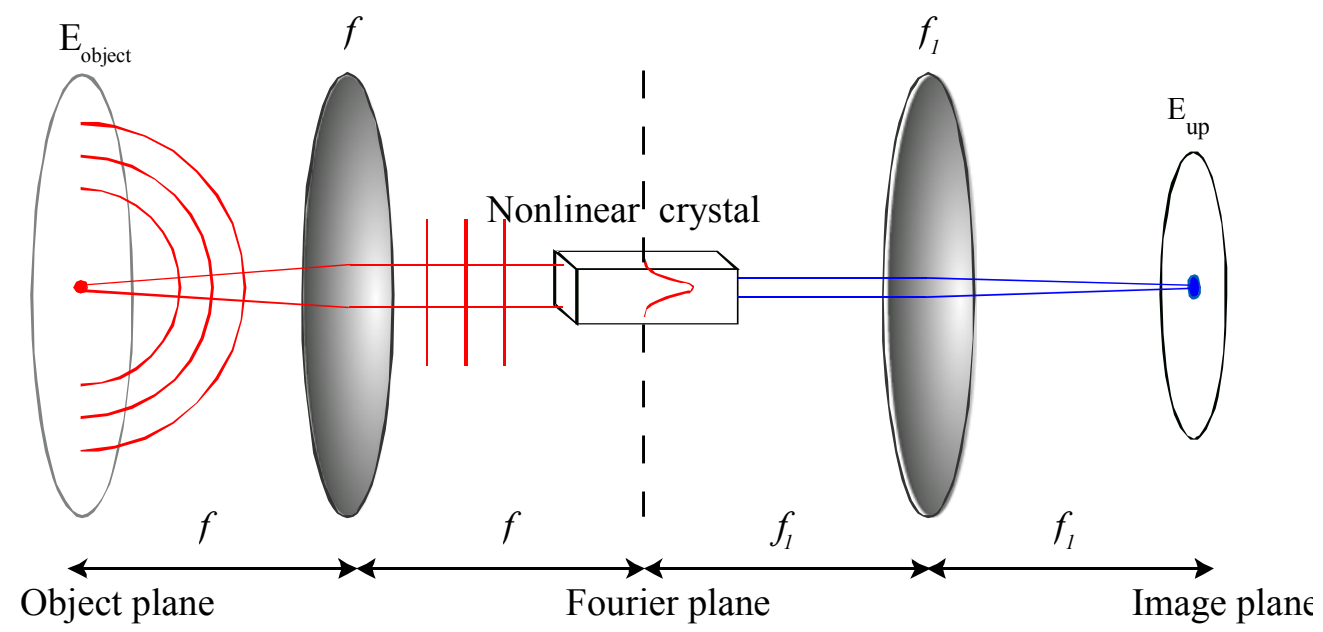

Figure 2. The upconversion of a point source located on the optical axis.

The imaging process can be explained as follows with reference to Figure 2. A point source located at the object plane is transformed into a plane wave at the Fourier plane using a first lens (f). The direction/angle of the plane wave is determined uniquely by the position of the point source at the object plane. Specifically for a point source located on the optical axis, as shown in Figure 2, the wave propagates along the z- axis. Assuming that the acceptance angle of the nonlinear upconversion process is sufficiently large compared to the angle of the incident plane wave, an upconverted photon may be generated during the passage of the SFG crystal (pp-KTP).Similarly, the wavelength of the emitted light from the point source should be within the bandwidth of the pp-KTP crystal. Figure 3 shows the actual acceptance angle and bandwidth of the presented configuration. The conversion efficiency or conversion probability is proportional to the circulating power of the $1342 \mathrm{~nm}$ beam as long as the efficiency is justified by the small signal approximation. The second lens $\left(f_{1}\right)$ transforms the upconverted plane wave into a single point at the image plane, however blurred by the finite size of the $1342 \mathrm{~nm}$ Gaussian beam waist at the Fourier plane. Note that also a magnification in the upconversion takes place since the upconverted wavelength light diffracts less than the light emitted from the object plane. A CCD camera suited for visible lights is then used to capture the images.
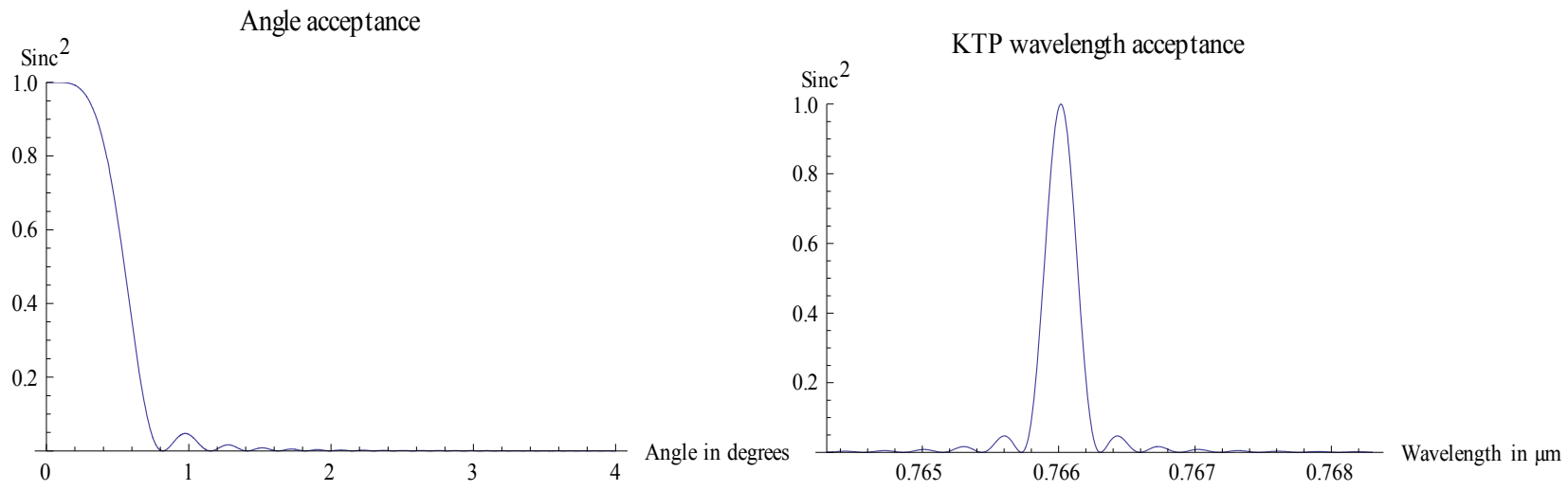

Figure 3. Left figure shows the acceptance angle, which defines an effective NA of the nonlinear upconversion system. Similarly the right figure shows the acceptance bandwidth of the upconversion system. 


\section{EXPERIMENTAL RESULTS AND DISCUSSION}

Figure 4 shows an upconverted image of a coiled thread (the light emitting filament) used in a standard light bulb. This image demonstrates that the imaging process has a good depth of field, since the coiled thread bends app. 60 degree out of focus, while the upconverted image remains reasonably sharp.

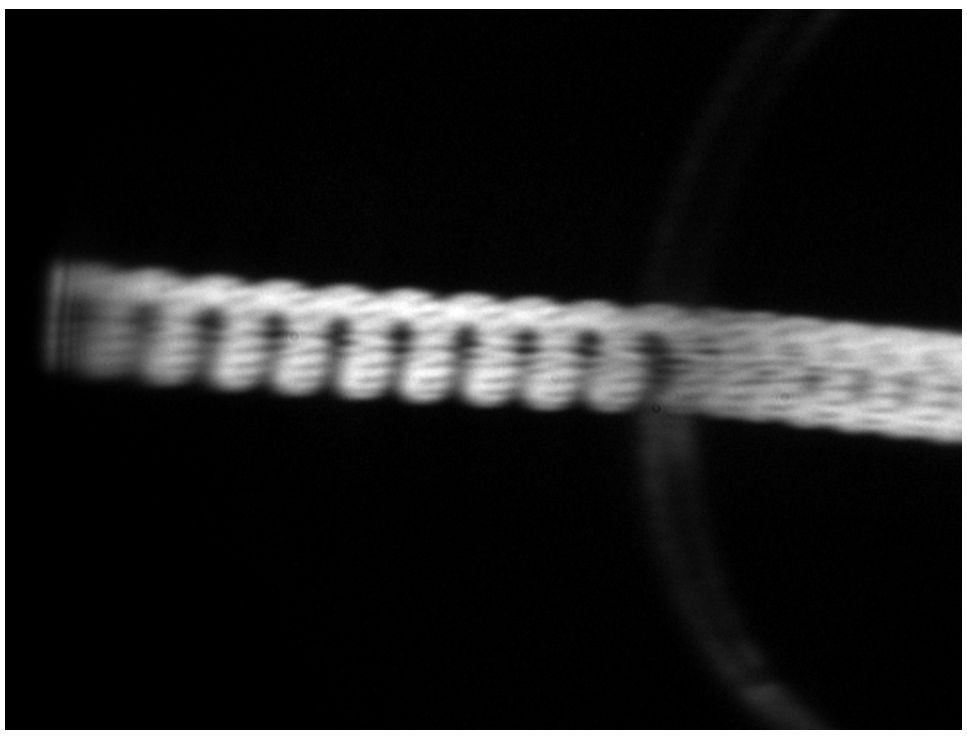

Figure 4. An upconverted image of a filament inside a light bulb. Note that the filament (a coiled thread) is bending out of the object plane near the right edge of the image.

The depth of focus in the object plane (aka depth of field) for an imaging system through a Gaussian aperture can be calculated as the corresponding confocal parameter in the object plane. If $f$ is the focal length of the first lens, and $w_{0}$ is the beam radius of the Gaussian mixing field, then the corresponding beam radius, $w_{0}^{\prime}$ in the object plane can be calculated to be:

$$
w_{0}{ }^{\prime}=\frac{\lambda f}{\pi w_{0}}
$$

The confocal parameter is defined to be:

$$
\text { Depth-of-field }=\frac{2 \pi w_{0}^{\prime 2}}{\lambda}=\frac{2 \pi}{\lambda}\left(\frac{\lambda f}{\pi w_{0}}\right)^{2}=\frac{2 \lambda f^{2}}{\pi w_{0}^{2}}
$$

The depth of field for the object is calculated by inserting $f=16 \mathrm{~mm}, w_{0}=600 \mu \mathrm{m}$, and $\lambda=766 \mathrm{~nm}$ to be $\sim 350 \mu \mathrm{m}$. This explains why the entire image in Fig. 4 appears in focus.

As seen from Figure $3 b$ the bandwidth of the upconversion system is app. $0.26 \mathrm{~nm}$. Thus the image is not only upconverted but also spectrally filtered. This feature can be used in several ways depending on the purpose, i.e. for low noise imaging in a small spectral window centered at specific line of a gas molecule. Note that noise outside the bandwidth of the nonlinear interaction process is suppressed. The Quantum efficiency is about $2 \times 10^{-4}$ in the present setup. The main reason for the smaller conversion efficiency compared to ref 4 relates to a un-optimized enhancement 
cavity as well as much larger beam radii. A low loss cavity design will allow us to reach in the order of 1-10\% quantum efficiency assuming a circulating $1342 \mathrm{~nm}$ power of about 150-200 Watts. Figure 5 shows the upconverted image from an incoherently illuminated resolution target. The resolution of the upconverted image is about $200 \times 1000$ pixels. See $\operatorname{ref}[5]$.
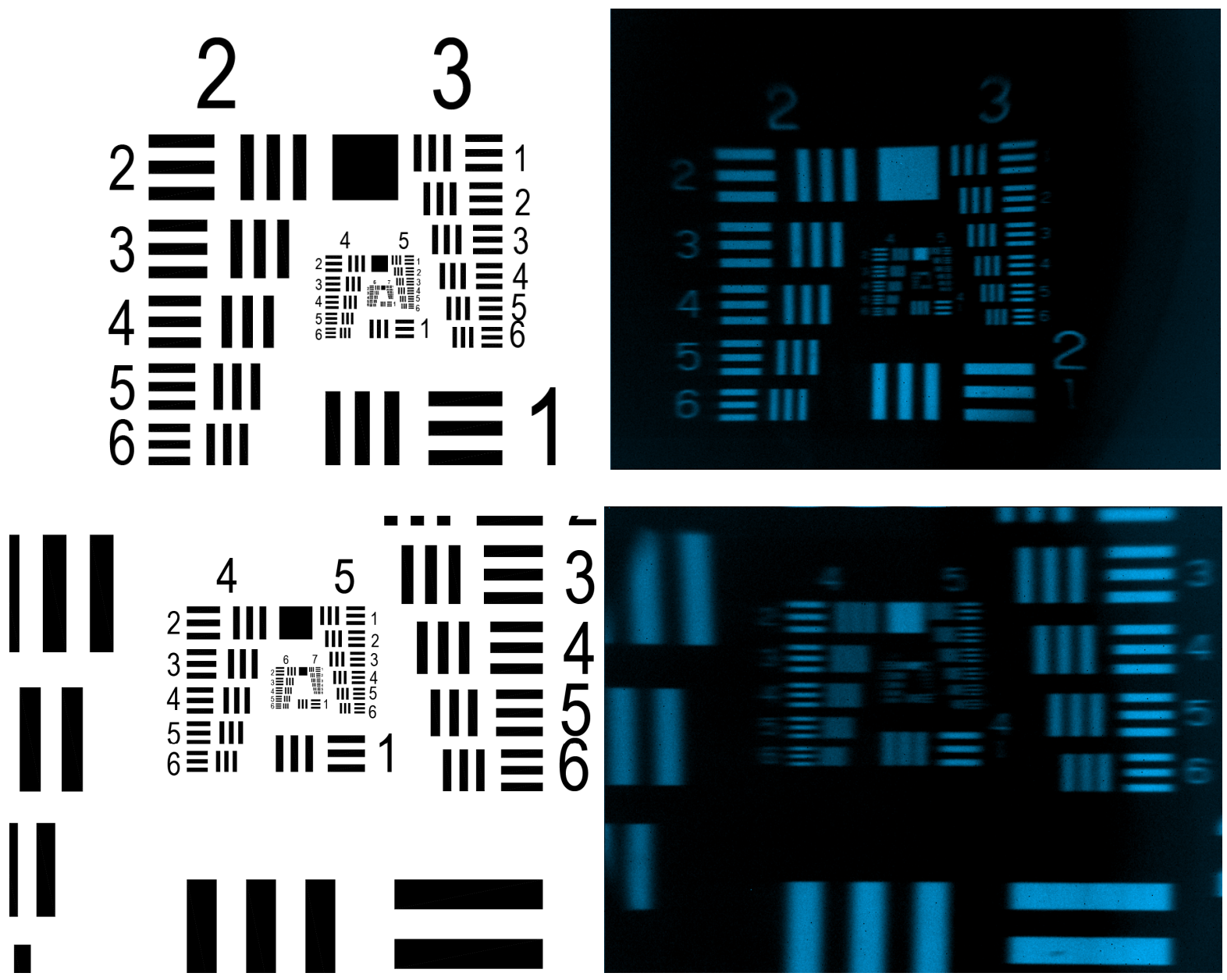

Figure 5. Upper left shows the high resolution transmission filter (resolution target). Upper right shows the upconverted image using the incoherently illuminated resolution target from upper left. The lower row shows the central parts of upper row images.

\section{CONCLUSION AND OUTLOOK}

We have demonstrated the concept of image upconversion of incoherent light. The combination of periodically poled crystals, intra-cavity enhancement and a simple 4-f design has proven to give a simple system with very good and predictable characteristics. A pictorial explanation of the upconversion process is outlined based on Huygens-Fresnel principle. We have upconverted incoherent light from the filament of an ordinary light bulb. Even objects out of focus are reasonably well imaged proving good depth of field. A quantum efficiency of $2 \times 10^{-4}$ was obtained - limited by the low circulating $1342 \mathrm{~nm}$ power. 1-10\% quantum efficiency should readily be obtained using a high finesse enhancement cavity. An upconverted image with a resolution of 200x1000 pixels was demonstrated. 


\section{REFERENCES}

[1] Midwinter, J. E. "Infrared up conversion in lithium-niobate with large bandwidth and solid acceptance angle," Appl. Phys. Lett. 14, 29-32 (1969).

[2] Falk, J. \& Tiffany, W. B. "Theory of parametric upconversion of thermal images," J. Appl. Phys. 43, 3762-3769 (1972).

[3] Lucy, R. F. “Infrared to Visible Parametric Upconversion,” Appl. Opt. 11, 1329-1336 (1972).

[4] Pedersen, C., Karamehmedović, E., Dam, J. S. \& Tidemand-Lichtenberg, P. "Enhanced 2D-image upconversion using solid-state lasers," Opt. Express 17, 20885-20890 (2009).

[5] Dam, J. S., Pedersen, C., Tidemand-Lichtenberg, P., "High-resolution two-dimensional image upconversion of incoherent light," Opt. Lett. 35, 3796-3798 (2010). 